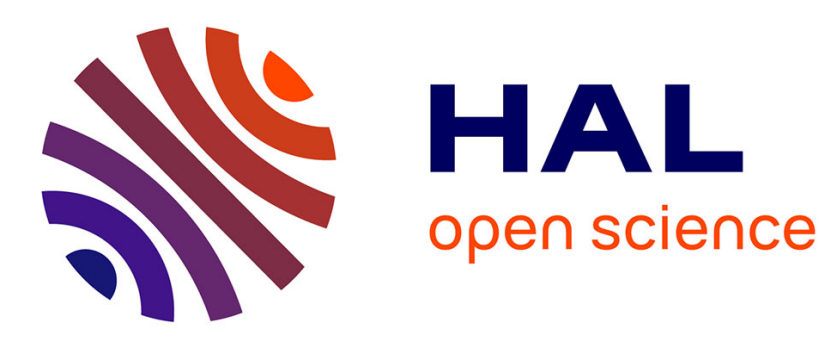

\title{
DCT-OFDM with Index Modulation
}

Marwa Chafii, Justin P Coon, Dene A Hedges

\section{To cite this version:}

Marwa Chafii, Justin P Coon, Dene A Hedges. DCT-OFDM with Index Modulation. IEEE Communications Letters, 2017, 21 (7), pp.1489-1492. 10.1109/LCOMM.2017.2682843 . hal-01488900

\section{HAL Id: hal-01488900 \\ https://hal-centralesupelec.archives-ouvertes.fr/hal-01488900}

Submitted on 14 Mar 2017

HAL is a multi-disciplinary open access archive for the deposit and dissemination of scientific research documents, whether they are published or not. The documents may come from teaching and research institutions in France or abroad, or from public or private research centers.
L'archive ouverte pluridisciplinaire HAL, est destinée au dépôt et à la diffusion de documents scientifiques de niveau recherche, publiés ou non, émanant des établissements d'enseignement et de recherche français ou étrangers, des laboratoires publics ou privés. 


\title{
DCT-OFDM with Index Modulation
}

\author{
Marwa Chafii, Member, IEEE, Justin P. Coon, Senior Member, IEEE, and Dene A. Hedges, Member, IEEE
}

\begin{abstract}
In this letter, index modulation (IM) is proposed for OFDM systems based on a discrete cosine transform (DCT) implementation. The new DCT-OFDM-IM scheme is shown to yield spectral efficiency improvements of up to $38 \%$ relative to OFDM-IM and up to $55 \%$ relative to OFDM while occupying the same bandwidth and maintaining similar robustness against frequency selective fading channels for low modulation orders.
\end{abstract}

Index Terms-Discrete cosine transform, orthogonal frequency division multiplexing, index modulation, spectral efficiency.

\section{INTRODUCTION}

The need for spectrally and energy efficient modulation schemes that will perform well in wideband channels and at the cell edge in future cellular systems has spurred on developments in so-called index modulation (IM) schemes [1]-[3]. These techniques, when applied to OFDM waveforms, yield the ability to encode information in an index set corresponding to active subcarriers as well as in the amplitudes and phases of those subcarriers. More recently, generalizations of OFDMIM have been proposed in an effort to yield higher spectral efficiencies [4] and better system performance [5]. Furthermore, OFDM-IM variants have been proposed as possible downlink solutions for cell-edge communication in 5G networks [6], [7].

Instead of using the discrete Fourier transform (DFT), as is typical for OFDM, a set of (co)sinusoidal functions has been proposed for multicarrier data transmission along with a discrete cosine transform (DCT) implementation [8], [9]. The resulting system, known as DCT-OFDM, requires only half the minimum subcarrier spacing needed for OFDM $^{1}$, thus doubling the number of subcarriers within the same total bandwidth. In this paper, we show that DCT-OFDM with IM is a promising technique for enhancing spectral efficiency compared with OFDM-IM. We describe the proposed scheme in Section II. Spectral efficiency is investigated in Section III, while Section IV contains results on the bit-error rate (BER) performance of the new method as well as relevant benchmarks. Conclusions are drawn in Section V.

\section{SyStem Description AND BENCHMARKS}

\section{A. DCT-OFDM}

In conventional OFDM, data is modulated using the complex basis of Fourier exponential functions defined as

$$
\phi_{n}(t)=\frac{1}{\sqrt{\mathcal{T}}} e^{j 2 \pi n \Delta_{\mathcal{F}} t}, 0 \leq t<\mathcal{T}, n \in \llbracket 0, \mathcal{N}-1 \rrbracket,
$$

M. Chafii is with CentraleSupélec/IETR, 35576 Cesson-Sévigné, France (email: marwa.chafii@supelec.fr).

J. P. Coon, and D. A. Hedges are with Department of Engineering Science, University of Oxford, Parks Road, Oxford, OX1 3PJ, UK (e-mail: justin.coon@eng.ox.ac.uk, dene.hedges@wadh.ox.ac.uk)

${ }^{1}$ We refer to DFT-based OFDM schemes as "OFDM" throughout the paper. where $\mathcal{N}$ is the number of subcarriers, $\mathcal{T}$ is the OFDM symbol period, and $\Delta_{\mathcal{F}}=1 / \mathcal{T}$ is the minimum intercarrier spacing required to satisfy the orthogonality constraint $\int_{0}^{\mathcal{T}} \phi_{n}(t) \phi_{n^{\prime}}(t) d t=0$ if $n \neq n^{\prime}$, with the integral evaluating to 1 if $n=n^{\prime}$. In DCT-OFDM, the following set of (co)sinusoidal functions is used for data modulation:

$$
\sigma_{n}(t)=\sqrt{\frac{2}{T}} \cos \left(2 \pi n \delta_{\mathcal{F}} t\right), 0 \leq t<T, n \in \llbracket 0, N-1 \rrbracket,
$$

where $N$ is the number of subcarriers, and $T$ is the DCT-OFDM symbol duration. Orthogonality is satisfied for a minimum intercarrier distance of $\delta_{\mathcal{F}}=1 /(2 T): \int_{0}^{T} \sigma_{n}(t) \sigma_{n^{\prime}}(t) d t=0$ if $n \neq n^{\prime}$, with the integral evaluating to 1 otherwise.

Let $\mathcal{W}(\mathrm{Hz})$ be the available channel bandwidth, and $T=\mathcal{T}$ be the symbol duration of both schemes. $\mathcal{W}$ can be occupied by $\mathcal{N}$ OFDM subcarriers spaced by $\Delta_{\mathcal{F}}$ or by $N$ DCT-OFDM subcarriers spaced by $\delta_{\mathcal{F}}$, so that $\Delta_{\mathcal{F}}=2 \delta_{\mathcal{F}}$, and $N=2 \mathcal{N}$. For DCT-OFDM, the bandwidth resources can be divided into $2 \mathcal{N}$ narrow subchannels instead of $\mathcal{N}$ compared with OFDM, while maintaining the perfect reconstruction condition.

The transmitted (DCT-)OFDM signals are given by

$$
\begin{aligned}
x_{\text {OFDM }}(t) & =\frac{1}{\sqrt{T}} \sum_{n=0}^{\mathcal{N}-1} C_{n} e^{j 2 \pi n t / T} \\
x_{\text {DCT-OFDM }}(t) & =\sum_{n=0}^{N-1} R_{n} \beta_{n} \cos (\pi n t / T),
\end{aligned}
$$

where $\beta_{n}=1 / \sqrt{T}$ if $n=0$ and $\beta_{n}=\sqrt{2 / T}$ otherwise. $C_{n}$ $\left(R_{n}\right)$ denotes an input symbol from a complex (real) $\mathcal{M}$-ary ( $M$-ary) constellation mapping, and modulated by subcarrier index $n$. The associated alphabet set is denoted by $\mathcal{S}_{\mathcal{M}}\left(S_{M}\right)$. We assume $M=\sqrt{\mathcal{M}}$ [9] since DCT-OFDM is a real signal.

\section{B. DCT-OFDM-IM}

Let a stream of $B$ input information bits be mapped to a DCT-OFDM-IM symbol of duration $T$. The $B$ bits are split into $G$ groups, each of $P$ bits $(B=P G)$. Each group of $P$ bits is mapped to a sub-block of length $N_{0}$ subcarriers. The total number of subcarriers is $N$, such that $N=G N_{0}$. Each subblock contains $N_{0}$ subcarriers spaced by $\Delta_{\mathcal{F}} / 2$. For each group $g$, only $K_{0}$ subcarriers out of $N_{0}$ are active. The total number of active subcarriers is $K=G K_{0}$ per $B$-bit transmission. A diagram of a DCT-OFDM-IM transmitter is shown in Fig. 1. We now briefly describe each transmitter block.

Bit splitter: $P$ bits of each group are divided into two subgroups with $P_{1}$ and $P_{2}$ bits each, such that $P=P_{1}+P_{2}$, with $P_{1}=\left\lfloor\log _{2}\left(\begin{array}{l}N_{0} \\ K_{0}\end{array}\right)\right\rfloor$ denoting the number of bits to be mapped 


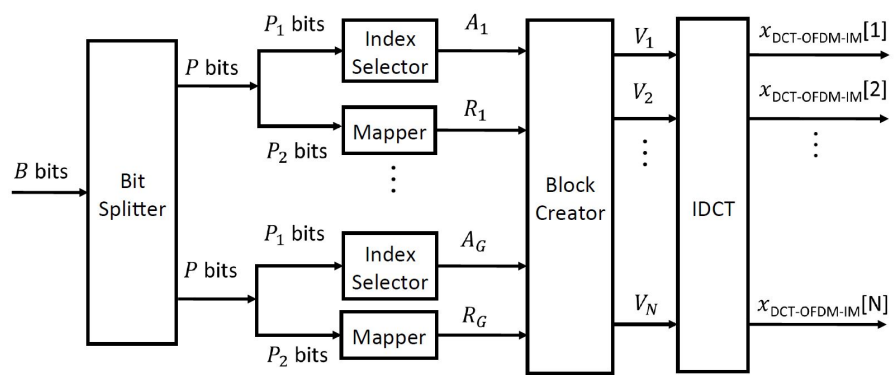

Fig. 1: DCT-OFDM-IM transmitter.

to an index set of active tones and $P_{2}=K_{0} \log _{2}(M)$ denoting the number of bits mapped to $M$-ary constellation symbols.

Index selector: By applying a selection procedure such as a look-up table or combinatorial mapping ${ }^{2}$, the $P_{1}$ bits for each group $g$ are mapped into an index set $A_{g}=\left\{a_{g, 1}, a_{g, 2}, \ldots, a_{g, K_{0}}\right\}$ of $K_{0}$ active subcarriers.

Constellation mapper: A real $M$-ary constellation is used for DCT-OFDM-IM (e.g., $M$-ary PAM).

Generation of IDCT input vector: Based on the index selector and mapper outputs, a vector $V=\left[V_{0}, V_{1}, \ldots, V_{N}\right]$ of length $N$ is created as follows:

$$
V_{n}= \begin{cases}R_{n}, & \text { if } n \in \bigcup_{g=1}^{G} A_{g} \\ 0, & \text { otherwise. }\end{cases}
$$

IDCT modulation: consists in applying an IDCT of length $N$ at the transmitter side (a DCT is performed for the demodulation at the receiver side). The transmitted DCT-OFDM-IM signal is therefore expressed as

$$
x_{\text {DCT-OFDM-IM }}(t)=\sum_{n=0}^{N-1} V_{n} \beta_{n} \cos (\pi n t / T) .
$$

\section{Other OFDM-IM Variants}

Conventional OFDM-IM employs a nearly identical principle to that of DCT-OFDM-IM, but where the complex field is used for modulation (i.e., the constellation mapper supports $\mathcal{M}$-ary QAM). We define the following notation for OFDM-IM: $\mathcal{B}$ is the number of input information bits, $\mathcal{G}$ is the number of groups, each of length $\mathcal{N}_{0}$ subcarriers and having $\mathcal{K}_{0}$ active subcarriers. $\mathcal{K}=\mathcal{G} \mathcal{K}_{0}$ is the total number of active subcarriers.

OFDM-IQ-IM is a variant of OFDM-IM that was proposed in [4]. Its principle consists of equally splitting the input bits dedicated for IM into two parts: one for in-phase components and one for quadrature components. The $\mathcal{M}$-ary constellation $(\mathcal{M}$-ary QAM) symbol is then constructed by combining the output of these two index modulations. Let $\mathcal{G}$ be the number of groups considered in OFDM-IQ-IM, each of length $\mathcal{N}_{0}$ subcarriers and having $\mathcal{K}_{0}^{\mathrm{IQ}}$ active subcarriers.

\footnotetext{
${ }^{2}$ See [3] for more information on these mapping techniques.
}

\section{Spectral Efficiency Analysis}

The key benefit that DCT-OFDM-IM offers relative to the aforementioned benchmarks is a higher spectral efficiency, which is defined as the rate (in bits per second) of communication in a given bandwidth. To explore this benefit, we assume here that DCT-OFDM, OFDM-IM, OFDM-IQ-IM are allocated the same channel bandwidth $\mathcal{W}$ and have the same symbol period $T$. Moreover, we assume that the number of groups considered in DCT-OFDM-IM is equal to that considered in OFDM-IM and OFDM-IQ-IM, i.e., $G=\mathcal{G}$, which gives us $N=2 \mathcal{N}$ and $N_{0}=2 \mathcal{N}_{0}$. Since the grouping is related to the detector complexity, this assumption is justified by the fact that for each group in OFDM-IQ-IM, the complexity is related to the detection of $2 N_{0}$ real symbols ( $N_{0}$ for in-phase symbols and $N_{0}$ for quadrature symbols), which is similar to the complexity needed for one group in DCT-OFDM-IM of length $2 N_{0}$. Moreover, OFDM-IM employs a complex constellation, and thus the detector complexity in this case is comparable to that of DCT-OFDM-IM and OFDM-IQ-IM under the constant group assumption noted above.

\section{A. Spectral Efficiency for Finite Numbers of Subcarriers}

$\mathcal{K}_{0}\left(\mathcal{K}_{0}^{\mathrm{IQ}}\right.$ resp.) tones are selected out of $\mathcal{N}_{0}$ for OFDM-IM (OFDM-IQ-IM resp.), and $\log _{2}(\mathcal{M})$ data bits are assigned to each selected tone. Knowing that the bandwidth $\mathcal{W}=\Delta_{\mathcal{F}} \mathcal{N}$, we can calculate the spectral efficiency for OFDM-IM and OFDM-IQ-IM to be

$$
\begin{aligned}
\xi_{\text {OFDM-IM }} & =\frac{G}{\mathcal{N}}\left(\left\lfloor\log _{2}\left(\begin{array}{c}
\mathcal{N}_{0} \\
\mathcal{K}_{0}
\end{array}\right)\right\rfloor+\mathcal{K}_{0} \log _{2}(\mathcal{M})\right) \\
\xi_{\text {OFDM-IQ-IM }} & =\frac{G}{\mathcal{N}}\left(2\left\lfloor\log _{2}\left(\begin{array}{c}
\mathcal{N}_{0} \\
\mathcal{K}_{0}^{\mathrm{IQ}}
\end{array}\right)\right\rfloor+\mathcal{K}_{0}^{\mathrm{IQ}} \log _{2}(\mathcal{M})\right) .
\end{aligned}
$$

Similarly, one can easily calculate the spectral efficiency of DCT-OFDM-IM to be

$$
\xi_{\text {DCT-OFDM-IM }}=\frac{G}{\mathcal{N}}\left(\left\lfloor\log _{2}\left(\begin{array}{l}
N_{0} \\
K_{0}
\end{array}\right)\right\rfloor+\frac{K_{0}}{2} \log _{2}(\mathcal{M})\right) .
$$

\section{B. Maximum Spectral Efficiency for Many Subcarriers}

In order to provide insight into the behaviour of the spectral efficiency, we explore the asymptotic regime (i.e., large number of subcarriers) to characterize the number of active subcarriers that maximizes the spectral efficiency. To make progress with the analysis, we ignore the floor function $\lfloor\cdot\rfloor$ in (7)-(9). Let $\mathcal{K}_{0}=\alpha \mathcal{N}_{0}, K_{0}=\beta N_{0}$, and $\mathcal{K}_{0}^{\mathrm{IQ}}=\gamma \mathcal{N}_{0}$, where $\alpha, \beta$, and $\gamma$ are parameters in the interval $[0,1]$. By using Stirling's approximation to simplify the binomial coefficients, it is easy to find asymptotic expressions for the spectral efficiencies of the three IM schemes. Taking derivatives of these expressions with respect to $\alpha, \beta$, and $\gamma$, and letting $\mathcal{N} \rightarrow \infty$, one can show that the spectral efficiency maximizing values are given by

$$
\alpha=\frac{\mathcal{M}}{\mathcal{M}+1} \quad \text { and } \quad \beta=\gamma=\frac{\sqrt{\mathcal{M}}}{\sqrt{\mathcal{M}}+1} .
$$

These maximizing proportions of active subcarriers are surprisingly accurate for finite numbers of subcarriers (cf. Fig. 2). 


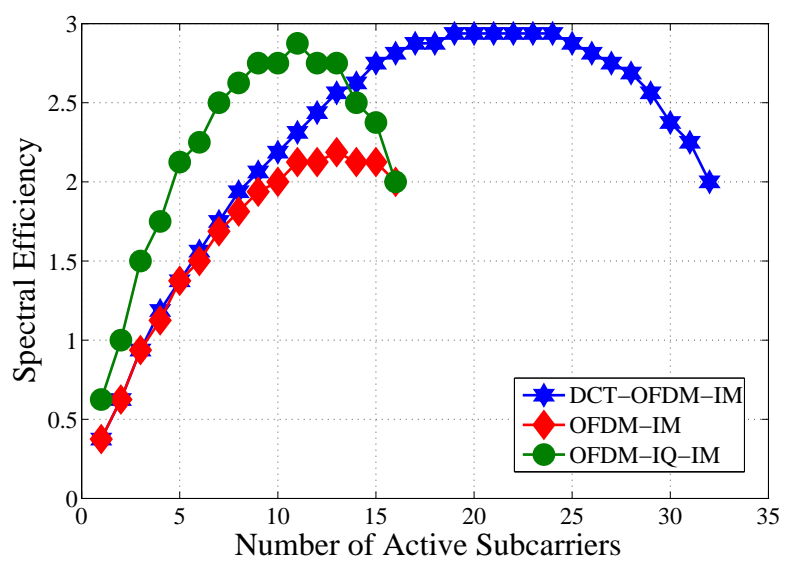

Fig. 2: Spectral efficiency vs. number of active subcarriers.

\section{Spectral Efficiency vs. Number of Active Subcarriers}

Fig. 2 shows the spectral efficiency of DCT-OFDM-IM for various numbers of active tones $K_{0} \in \llbracket 1,32 \rrbracket$, and that of OFDM-IM and OFDM-IQ-IM for $\mathcal{K}_{0}, \mathcal{K}_{0}^{\mathrm{IQ}} \in \llbracket 1,16 \rrbracket$. The comparison is performed for $\mathcal{M}=4, \mathcal{N}=16$, and $G=1$. An initial observation of the three curves illustrates the potential for spectral efficiency improvements offered by DCT-OFDMIM. It is also observed from Fig. 2 that the maximum spectral efficiency of OFDM-IQ-IM is similar to that of DCT-OFDMIM in this example. However, it should be noted that this is an artefact that results when we consider a single group. Dividing the input information bits into $G>1$ groups will result in reduced peak spectral efficiency for the three IM schemes discussed here; however, this approach must be employed to reduce implementation complexity, which can be prohibitively high for large $N$ due to the combinatorial nature of IM techniques. As we increase $G$, we will see below that the peak spectral efficiency of DCT-OFDM-IM is consistently higher than that of the OFDM-IQ-IM scheme.

\section{Spectral Efficiency vs. Number of Groups}

To compare the spectral efficiencies of the IM schemes against non-IM benchmarks, we let $\alpha, \beta$, and $\gamma$ satisfy (10) and set $N=128$. We define the spectral efficiency increase of technique $x$ relative to $y$ as

$$
\rho_{x / y}=\frac{\xi_{\mathrm{x}}-\xi_{\mathrm{y}}}{\xi_{\mathrm{y}}} .
$$

In Tab. I, a spectral efficiency comparison between different modulation schemes is presented ${ }^{3}$. We observe that the spectral efficiency of the different IM schemes is markedly increased relative to (DCT-)OFDM for low-modulation orders (more than $55 \%$ for $(M=2, G=1)$ when using DCT-OFDM-IM).

\footnotetext{
${ }^{3}$ Here, we ignore the guard interval. When considering the same length of the guard interval for all schemes, the absolute spectral efficiency for each will decrease, but the relative spectral efficiency will remain the same.
}

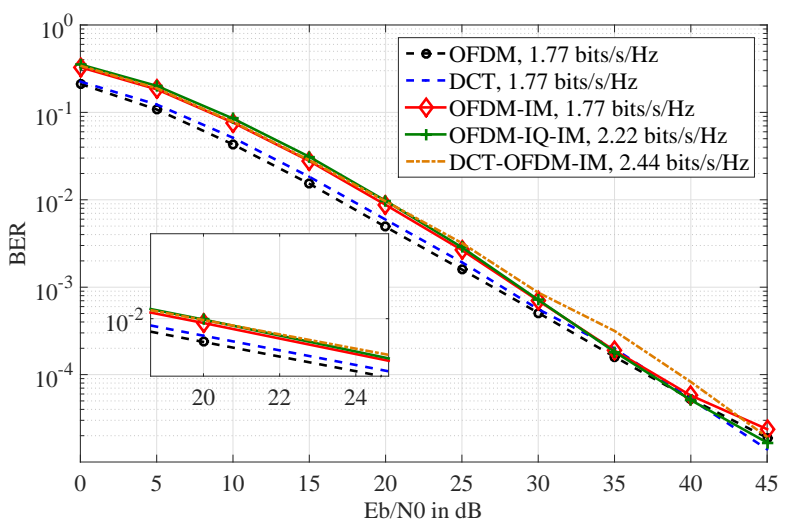

Fig. 3: BER performance comparison $(M=2, \mathcal{M}=4)$.

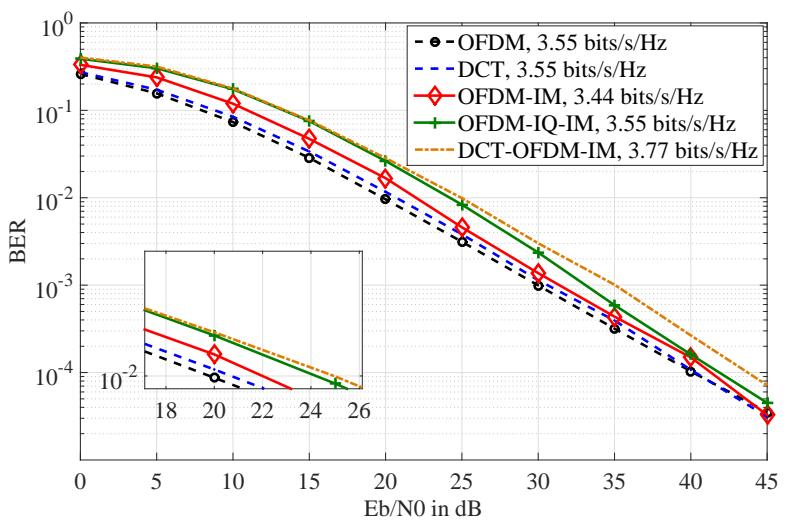

Fig. 4: BER performance comparison $(M=4, \mathcal{M}=16)$.

The gain is less significant for higher order constellations. Interestingly, although OFDM-IQ-IM exhibits good spectral efficiency gains relative to most other benchmarks, the proposed DCT-OFDM-IM scheme has the highest spectral efficiency of all schemes, even for the case where the DFT-based methods exploit 16-QAM. These observations strongly motivate further study of DCT-OFDM-IM variants.

\section{BER PERFORMANCE}

The BER performance of multicarrier schemes with IM depends on the detection method employed. Several schemes for index set detection have been reported in the literature [3]. Here, for a fair comparison of the studied schemes, a frequency domain equalizer using zero forcing (ZF) inversion of the channel is performed for the received signal (additional FFT/IFFT operations are required for DCT-OFDM-IM scheme), and a minimum distance detection is used to recover the index set and the transmitted symbols.

Here, the BER performance of DCT-OFDM-IM is shown in a frequency selective fading channel and compared with OFDM-IM and OFDM-IQ-IM using the following simulation parameters: $N=128, G=8, \Delta_{\mathcal{F}}=15 \mathrm{kHz}$ and $\alpha, \beta, \gamma$ satisfy (10). The channel used in this comparison is the extended 
Table I: Spectral efficiency comparison for $N=128$.

\begin{tabular}{|c||c|c|c|c||c|c|c|c|}
\hline \multicolumn{1}{|c|}{ Modulation Order } & \multicolumn{3}{|c||}{$M=2, \mathcal{M}=4$} & \multicolumn{4}{c|}{$M=4, \mathcal{M}=16$} \\
\hline number of groups $G$ & 1 & 2 & 4 & 8 & 1 & 2 & 4 & 8 \\
\hline$\xi_{\text {DCT-OFDM-IM }}$ & 3.1 & 3.06 & 2.93 & 2.75 & 4.57 & 4.53 & 4.43 & 4.25 \\
\hline$\xi_{\text {OFDM-IM }}$ & 2.26 & 2.21 & 2.12 & 2 & 4.04 & 4 & 4 & 3.87 \\
\hline$\xi_{\text {OFDM-IQ-IM }}$ & 3.06 & 2.93 & 2.75 & 2.5 & 4.53 & 4.43 & 4.25 & 4 \\
\hline$\xi_{\text {OFDM }}=\xi_{\text {DCT-OFDM }}$ & \multicolumn{7}{|c|}{2} & \multicolumn{5}{|c|}{4} \\
\hline$\rho_{\text {DCT-OFDM-IM/OFDM-IM }}$ & $37.24 \%$ & $38.02 \%$ & $38.23 \%$ & $37.5 \%$ & $13.13 \%$ & $13.28 \%$ & $10.94 \%$ & $9.68 \%$ \\
\hline$\rho_{\text {DCT-OFDM-IM/OFDM-IQ-IM }}$ & $1.53 \%$ & $4.25 \%$ & $6.81 \%$ & $10 \%$ & $1.03 \%$ & $2.11 \%$ & $4.41 \%$ & $6.25 \%$ \\
\hline$\rho_{\text {DCT-OFDM-IM /(DCT-OFDM, OFDM) }}$ & $55.46 \%$ & $53.12 \%$ & $46.87 \%$ & $37.50 \%$ & $14.45 \%$ & $13.28 \%$ & $10.93 \%$ & $6.25 \%$ \\
\hline$\rho_{\text {OFDM-IM /(DCT-OFDM, OFDM) }}$ & $13.28 \%$ & $10.93 \%$ & $6.25 \%$ & $0 \%$ & $1.17 \%$ & $0 \%$ & $0 \%$ & $-3.13 \%$ \\
\hline$\rho_{\text {OFDM-IQ-IM /(DCT-OFDM, OFDM }}$ ) & $53.12 \%$ & $46.87 \%$ & $37.5 \%$ & $25 \%$ & $13.28 \%$ & $10.94 \%$ & $6.25 \%$ & $0 \%$ \\
\hline
\end{tabular}

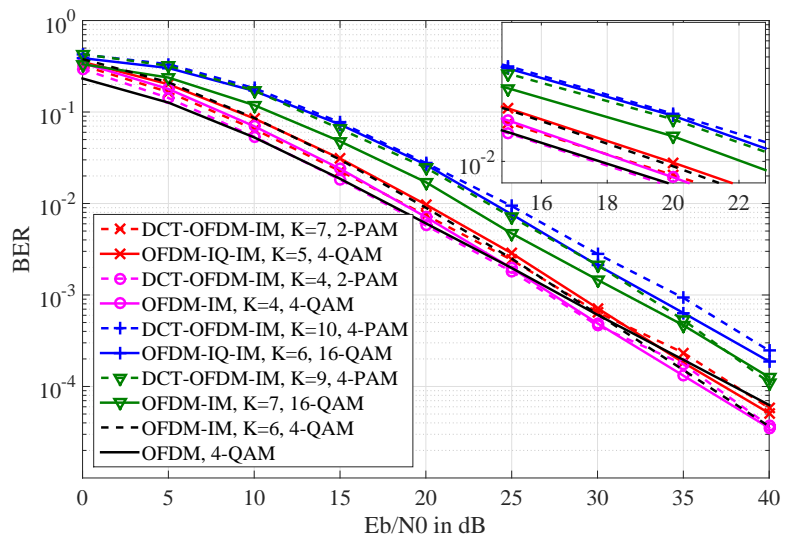

Fig. 5: BER performance comparison for the same spectral efficiency.

typical urban (ETU) model of the LTE standard [10]. A cyclic prefix is added to the transmitted signals of the studied schemes. A comparison of the BERs for the three schemes is shown in Fig. 3 for $(M=2, \mathcal{M}=4)$ and in Fig. 4 for $(M=4, \mathcal{M}=16)$. For low modulation order (Fig. 3), all three IM schemes exhibit similar performance, with the DCT scheme performing slightly worse at high $E_{b} / N_{0}$. One possible interpretation of this marginal loss is that the index detection errors are more likely to occur when the number of possible combinations is high. For high modulation order (Fig. 4), DCTOFDM-IM and OFDM-IQ-IM perform worse than OFDMIM. Note, however, that the spectral efficiency of the DCTbased scheme is higher than all other schemes (cf. Tab. I), which raises a trade-off between spectral efficiency and BER performance.

In Fig. 5, different schemes are compared pairwise (DCTOFDM-IM vs. OFDM-IM, DCT-OFDM-IM vs. OFDM-IQIM, and OFDM-IM vs. OFDM) under the constraint that they have the same spectral efficiency. We observe that, for low modulation order, DCT-OFDM-IM with K=7 (DCT-OFDM-IM, $\mathrm{K}=4$ resp.) slightly outperforms OFDM-IQ-IM (OFDM-IM, $\mathrm{K}=4$ resp.) for the same spectral efficiency of 2.22 (1.56 resp.) $\mathrm{bits} / \mathrm{s} / \mathrm{Hz}$. As previously mentioned, DCT-OFDM-IM BER performance is sensitive to high modulation order, while OFDM-
IM and OFDM-IQ-IM are not particularly useful when highorder constellations are applied since they do not achieve any significant gain, in terms of spectral efficiency or performance, relative to OFDM. Moreover, the DCT-OFDM-IM detection scheme is linear, not optimal. Also, no forward error correction is employed, and optimal index sets are not chosen. Hence, there is a lot of scope for improvement. These observations again motivate further study on DCT-OFDM-IM, particularly with respect to enhanced receiver design.

\section{CONCLUSION}

In this paper, we proposed DCT-OFDM with IM, and showed that it can achieve significant spectral efficiency gains relative to relevant benchmarks. In fading wireless channels, the new technique yields comparable BER performance to these benchmarks when minimum distance detection is employed along with ZF channel equalization. It is hoped that the potential of DCTOFDM-IM, which was highlighted herein, will motivate further research on DCT-based IM techniques, particularly with respect to receiver design and operation with high-order constellations, with a view to enhancing next generation systems.

\section{REFERENCES}

[1] R. Padovani and J. Wolf, "Coded phase/frequency modulation," IEEE Trans. Commun., vol. 34, no. 5, pp. 446-453, 1986.

[2] P. K. Frenger and N. A. B. Svensson, "Parallel combinatory OFDM signaling," IEEE Trans. Commun., vol. 47, no. 4, pp. 558-567, 1999.

[3] E. Başar, Ü. Aygölü, E. Panayırc1, and H. V. Poor, "Orthogonal frequency division multiplexing with index modulation," IEEE Trans. Signal Process., vol. 61, no. 22, pp. 5536-5549, 2013.

[4] R. Fan, Y. J. Yu, and Y. L. Guan, "Generalization of orthogonal frequency division multiplexing with index modulation," IEEE Trans. Wireless Commun., vol. 14, no. 10, pp. 5350-5359, 2015.

[5] Y. Xiao, S. Wang, L. Dan, X. Lei, P. Yang, and W. Xiang, "OFDM with interleaved subcarrier-index modulation," IEEE Commun. Lett., vol. 18, no. 8, pp. 1447-1450, 2014.

[6] S. Hong, M. Sagong, C. Lim, K. Cheun, and S. Cho, "FQAM: A modulation scheme for beyond $4 \mathrm{G}$ cellular wireless communication systems," in 2013 IEEE Globecom Workshops. IEEE, 2013, pp. 2530.

[7] E. Başar, "Index modulation techniques for $5 \mathrm{G}$ wireless networks," IEEE Commun. Mag., vol. 54, no. 7, pp. 168-175, 2016.

[8] J. Tan and G. L. Stuber, "Constant envelope multi-carrier modulation," in MILCOM 2002. Proceedings, vol. 1. IEEE, 2002, pp. 607-611.

[9] F. Xiong, "M-ary amplitude shift keying OFDM system," IEEE Trans. Commun., vol. 51, no. 10, pp. 1638-1642, 2003.

[10] T. ETSI, "136 101 v8. 23.0 (3gpp ts 36.101 v8. 23.0 release 8)." 